\title{
Nd:YAG Laser for Ahmed Tube Shunt Blockage in Patients Implanted with Boston Type I Keratoprosthesis
}

Jianjun Gu • Yuying Zhang · Jiajie Zhai · Zhancong Ou •

Jiaqi Chen (iD

Received: March 11, 2019 / Published online: April 29, 2019

(C) The Author(s) 2019

\section{ABSTRACT}

Introduction: The purpose of this study was to describe the results using the Nd:YAG laser to reopen blocked glaucoma tube shunts in three ocular chemical burn patients with Boston keratoprostesis type I (KPro) implantation.

Methods: The medical records of the three patients at Zhongshan Ophthalmic Centre were reviewed.

Results: Patient 1, who had glaucoma secondary to KPro implantation, had undergone Ahmed glaucoma valve (AGV) implantation to control an elevated intraocular pressure (IOP). One day after surgery, the tube was observed to

Jianjun Gu and Yuying Zhang contributed equally to this work, and both are considered principal authors.

Enhanced digital features To view enhanced digital features for this article go to https://doi.org/10.6084/ m9.figshare.7976390.

Electronic supplementary material The online version of this article (https://doi.org/10.1007/s40123019-0186-6) contains supplementary material, which is available to authorized users.

J. Gu $\cdot$ Y. Zhang $\cdot$ Z. Ou $\cdot$ J. Chen $(\bowtie)$

State Key Laboratory of Ophthalmology, Zhongshan

Ophthalmic Center, Sun Yat-sen University,

Guangzhou 510060, China

e-mail: docjiaqi@163.com

J. Gu $\cdot$ J. Zhai $\cdot$ J. Chen

Guangdong Visual and Ophthalmology Research

Institute, Guangzhou 510060, China be embedded in the residual lens capsule. The capsule was opened by one $1.5 \mathrm{~mJ}$ laser pulse, with a subsequent drop in the IOP. In patient 2, the AGV and KPro had been implanted simultaneously. One month after surgery, the IOP increased to $35 \mathrm{mmHg}$, estimated by palpation, and a vitreous gel was seen blocking the tube. A Nd:YAG laser pulse was used to open the occluded tube. In patient 3 , the tube was blocked by iris tissue; Nd:YAG laser treatment opened the tube.

Conclusions: The cases described here indicate that Nd:YAG laser treatment seems to be a valuable option for opening an occluded AGV tube in patients with KPro implantation. The correct location of the tube tip, visualized through the KPro optic, is essential for laser treatment.

Keywords: Boston; Keratoprosthesis; Glaucoma drainage device; Nd:YAG laser; Tube obstruction

\section{INTRODUCTION}

Boston type I keratoprosthesis (KPro; Massachusetts Eye and Ear Infirmary, Boston, MA, USA) is considered to be a viable treatment for various corneal diseases, including repeated graft failure, ocular chemical burns and stem cell deficiency. However, sustained intraocular pressure (IOP) elevation after KPro implantation places visual function in jeopardy due to the 
potential for optic nerve damage. An additional concern associated with high IOP after KPro implantation is the mechanical stress placed on the corneal carrier, which may result in ectasia. The major risk factors for the development of post-KPro glaucoma are pre-existing glaucoma, aphakia and anterior synechiae. Chronic inflammation is also associated with a high IOP. Glaucoma drainage device (GDD) surgery is an effective option to treat medically uncontrollable glaucoma after KPro implantation, although various complications are associated with this surgery, including tube exposure, tube occlusion and plate exposure and encapsulation [1]. Here, we describe the use of the neodymium (Nd):YAG laser to open occluded Ahmed glaucoma valve (AGV; New World Medical, Inc., Rancho Cucamonga, CA, USA) tubes to restore aqueous flow and control IOP in three patients with severe ocular chemical burns who had undergone KPro implantation.

\section{METHODS}

\section{Design}

This was a retrospective case series. The files of three patients (three eyes) with KPro implantation were reviewed. All patients had suffered severe ocular chemical burns and had been treated with Nd:YAG laser pulse(s) to open occluded AGV tubes.

This retrospective analysis was approved by the institutional ethical committee of Zhongshan Ophthalmic Centre. All procedures followed were in accordance with the Declaration of Helsinki of 1964, as revised in 2013. All patients provided written informed consent for publication of clinical data.

\section{CASE REPORT}

\section{Case 1}

A 52-year-old man presented to our hospital with decreased vision in both eyes due to ocular chemical burns (lime) in 2018. He had undergone lamellar keratoplasty in the left eye 2 years prior. On examination, his visual acuity (VA) in both eyes was hand motions with accurate light perception. Slit-lamp examination revealed total corneal opacification with pannus in the right eye. The left eye showed a failed large lamellar corneal button with $360^{\circ}$ corneal vascularization. No underlying anterior chamber details were visualized. Anterior segment ultrasound (ultrasound biomicroscopy, UBM) revealed an eye with a shallow anterior chamber depth and $180^{\circ}$ peripheral anterior synechiae. IOP estimated by digital palpation was in the normal range. Ultrasound imaging showed no obvious optic nerve cupping. After discussion with the patient, it was decided to proceed with KPro placement in the left eye in March 2018. His cataract was removed at the time of surgery by anterior vitrectomy, and an aphakic KPro was placed. At postoperative week 4, he had 10/100 vision with a cup disc ratio of 0.5 (Fig. 1a). At month 2, he started to develop glaucoma de novo, with a decrease in vision to counting fingers (FC) at $2 \mathrm{~m}$. His IOP was uncontrolled with topical and systemic anti-glaucoma medication, and he therefore had an AGV placement approximately 3 months after the KPro implantation. The shunt tube was introduced into the anterior chamber through a tract created by a 23-gauge syringe needle. The tip of the tube is long enough to be seen through the KPro optic. At postoperative day 1, the patient's IOP was $35 \mathrm{mmHg}$, estimated by digital palpation, and the tip of the tube was found to be embedded in the residual lens capsule (Fig. 1b). This situation was managed with one Nd:YAG laser pulse of $1.5 \mathrm{~mJ}$ to the membrane at the tube tip, which ruptured the membrane (Fig. 1c). The membrane retracted posteriorly, and the IOP dropped to $15 \mathrm{mmHg}$, estimated by palpation. Topical steroids were used to control the mild postoperative inflammation. To date, the IOP has remained low for 7 months. At the last follow-up, his visual acuity was FC at 1 inch with a cup disc ratio of 0.9 (Fig. 1d).

\section{Case 2}

A 51-year-old man who presented to our hospital with a diagnosis of ocular chemical burns 


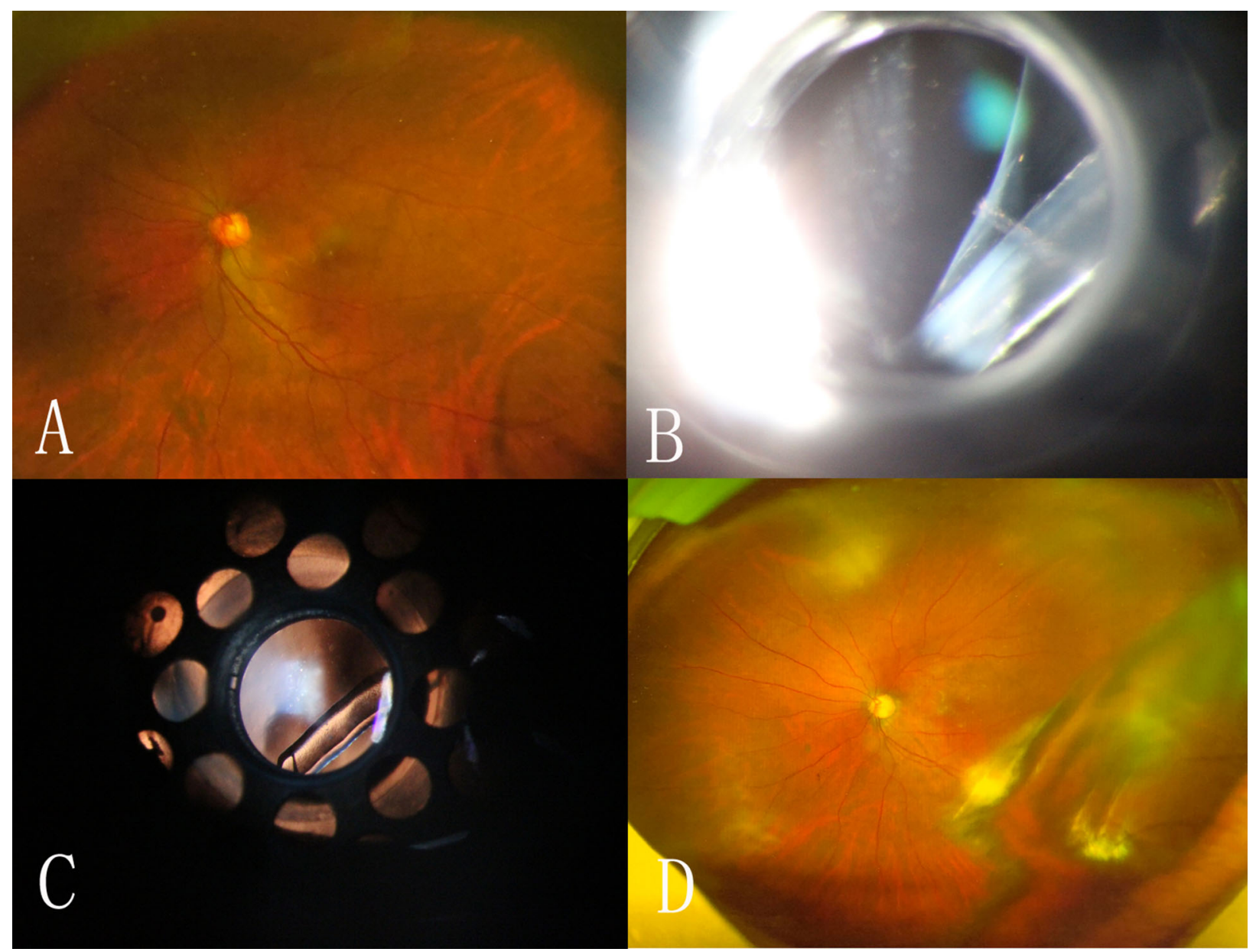

Fig. 1 a One month after Boston keratoprosthesis (KPro) implantation, laser retina scan image (Optomap ${ }^{\circledR}$, Optomap 200Tx ${ }^{\mathrm{TM}}$, Optos ${ }^{\circledR}$, Marlborough, MA, USA) of the left eye revealed a cup disc ratio of 0.5 . b Slit-lamp photograph of the left eye showing the tube was covered

had been injured by exposure to sodium hydroxide 13 months prior. He was examined at the Zhongshan Ophthalmic Centre Corneal Service in July 2018. He had a VA of hand motions in the right eye, and no light perception in the left eye. The IOP of the right eye, with maximal anti-glaucoma drugs, was $30 \mathrm{mmHg}$, estimated by digital palpation. Ocular examination revealed $360^{\circ}$ corneal vascularization and total corneal opacification (Fig. 2a). UBM showed $360^{\circ}$ peripheral anterior synechiae and iris-cornea touch. B-scan ultrasonography revealed the patient was phakic with attached retina, vitreous debris and glaucomatous cupping of the optic disc. The left eye with a residual lens capsule. c The occluding capsule was perforated by a Nd:YAG laser pulse of $1.5 \mathrm{~mJ}$. d Optomap ${ }^{\circledR}$ image demonstrating glaucoma drainage implant tube in the left eye with a cup disc ratio of 0.9

was phthisical and remarkable for extensive symblephara. As penetrating keratoplasty was doomed to fail, it was decided to implant a KPro. KPro placement with combined extracapsular cataract extraction, open-sky anterior vitrectomy and AGV implantation was performed in September 2018. By postoperative day 7, the patient's VA was FC. His IOP was approximately $15 \mathrm{mmHg}$, estimated by palpation. One month after surgery, he presented to us with a 3-day history of increasing pain in the right eye. His IOP was approximately $35 \mathrm{mmHg}$, estimated by palpation. Slit-lamp examination revealed the internal tube ostium was blocked by vitreous with pigment deposited in it 


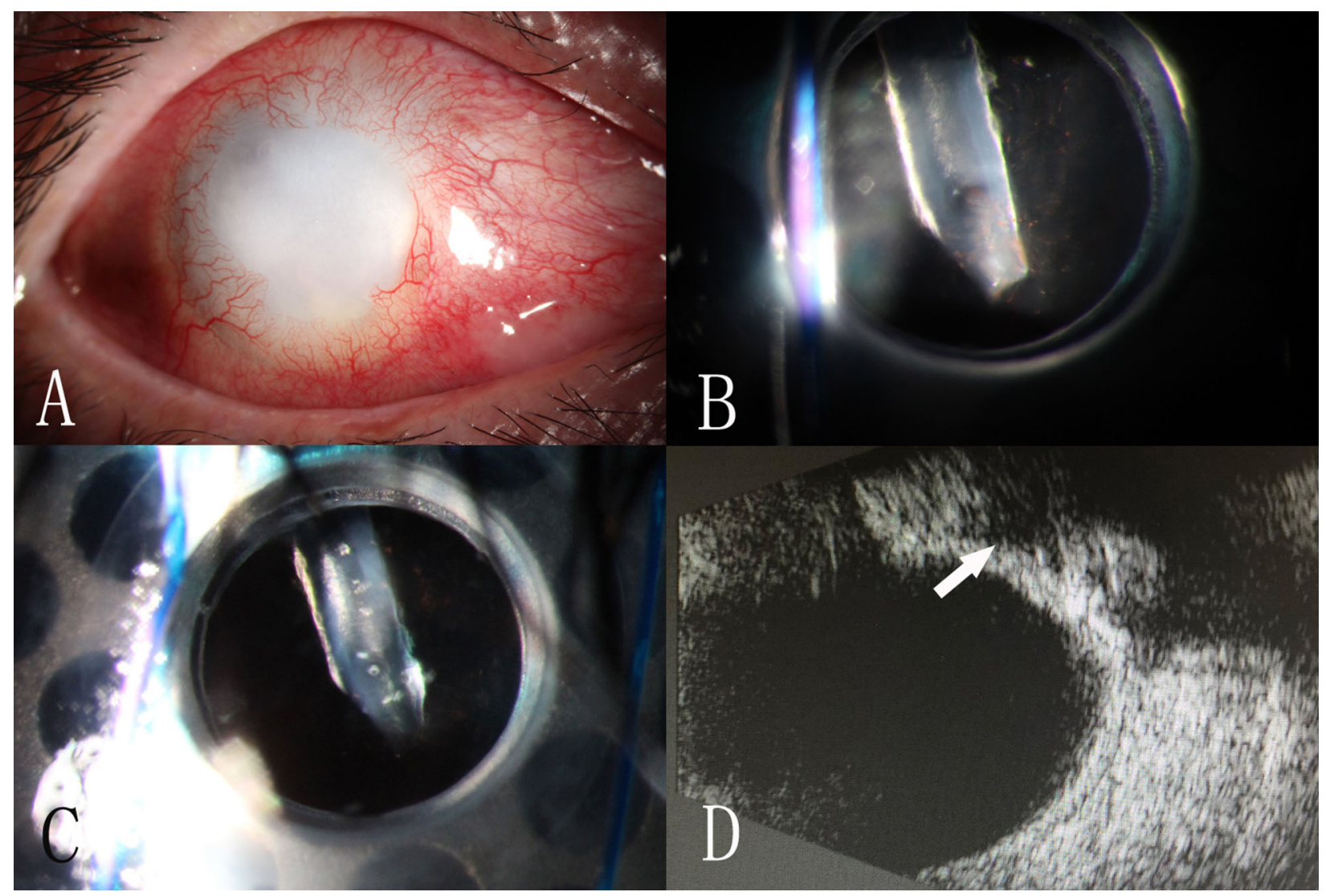

Fig. 2 a Slit-lamp image showing total limbal stem cell deficiency and opaque cornea of the right eye before surgery. b The tube orifice was blocked with translucent vitreous gel with pigment deposited in it. c The tube was

(Fig. 2b). The patient was treated with four Nd:YAG laser pulses of $1.8 \mathrm{~mJ}$ at the tube tip, creating a full-thickness hole in the vitreous and posterior retraction of the vitreous. Fluid turbulence followed immediately, and a piston-like movement of the micro air bubble in the tube was observed (Fig. 2c; see also video in the Electronic Supplementary Material). The IOP dropped to $15 \mathrm{mmHg}$, and the ocular pain was relieved. To date, the IOP has remained low for 3 months, and B-scan ultrasound demonstrated a fluid-filled reservoir surrounding the GDD plate (Fig. 2d).

\section{Case 3}

A 49-year-old man presented with total limbal stem cell deficiency and opaque cornea (Fig. 3a) opened by the Nd:YAG laser; a micro air bubble was created by the laser beam within the tube. d B-scan ultrasound showing echolucent fluid areas around the glaucoma drainage device plate (arrow)

following a chemical burn (cement) 3 years prior. UBM showed $270^{\circ}$ peripheral anterior synechiae and iris-cornea touch. He was managed with glaucoma drainage implant surgery combined with pars plana vitrectomy and KPro implantation [2]. At postoperative day 1, the tip of the tube was found to be obstructed with iris tissue (Fig. 3b). He was managed with two $\mathrm{Nd}$ :YAG laser two pulses of $1.5 \mathrm{~mJ}$ each to the iris at the tube tip. After surgery, the IOP remained low, and the tube stayed patent (Fig. 3c, d).

\section{DISCUSSION}

Glaucoma is the single most important factor limiting patients' visual outcomes after ocular 


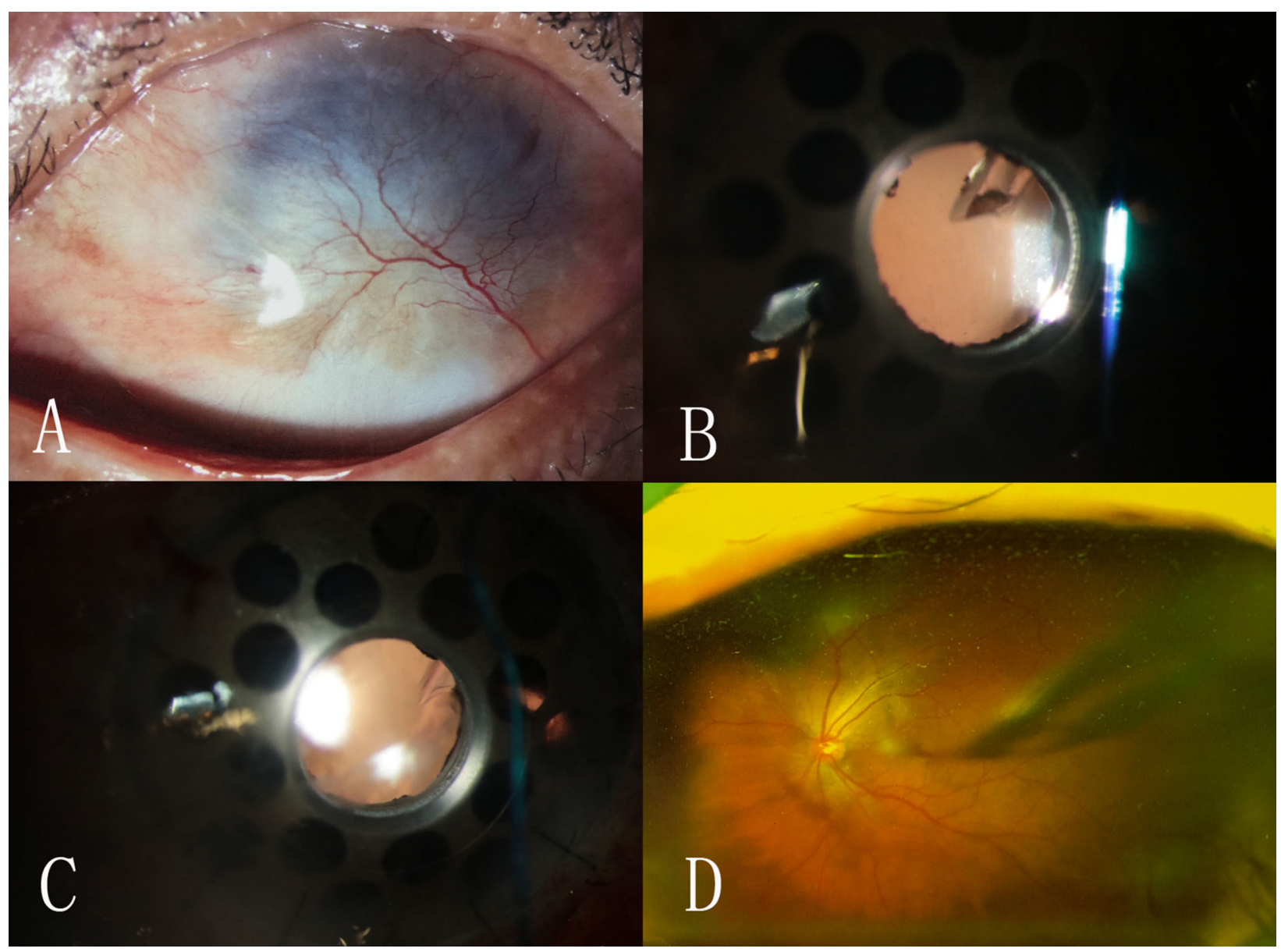

Fig. 3 a Slit-lamp image showing total limbal stem cell deficiency and an opaque cornea of the left eye before surgery. $\mathbf{b}$ The tube orifice was blocked with an iris tissue.

chemical burns [3]. Eyes with severe chemical burns are more likely to have glaucoma and require surgery to treat this condition [4]. Glaucoma is not uncommon after KPro surgery [5]. Treatment of post-KPro glaucoma involves the use of topical and systemic glaucoma medication. The disadvantage of topical therapy is inadequate drug penetrance through the central anterior optic plate. Chronic use of systemic medicine, such as oral carbonic anhydrase inhibitors, is associated with potential systemic effects, including metabolic acidosis and urolithiasis. GDD surgery has been recommended by the manufacturer of the KPro in eyes with pre-existing glaucoma [6].

The most common complication after GDD surgery is obstruction of the tube [7]. The tube c The tube was opened by treatment with a Nd:YAG laser pulse. $\mathbf{d}$ Optomap ${ }^{\circledR}$ image showing a patent tube

may be blocked by inflammatory tissue, vitreous gel and/or iris tissue [1]. Given the disadvantages of time-domain anterior segment optical coherence tomography (AS-OCT), including lower image definition and no transmission through metal (titanium) or the iris, we found it difficult to visualize the membrane or vitreous gel on the tip of the tube using ASOCT. The early diagnosis and treatment of these three patients relied on adequate visualization of the sufficiently long tube through the KPro optic by slit-lamp examination, the tube tip being cut bevelled up, good optical clarity of the anterior plate and less formation of a retroprosthetic membrane with titanium backplate. Severe ocular chemical burns are characterized by protracted and relentless inflammation, and 


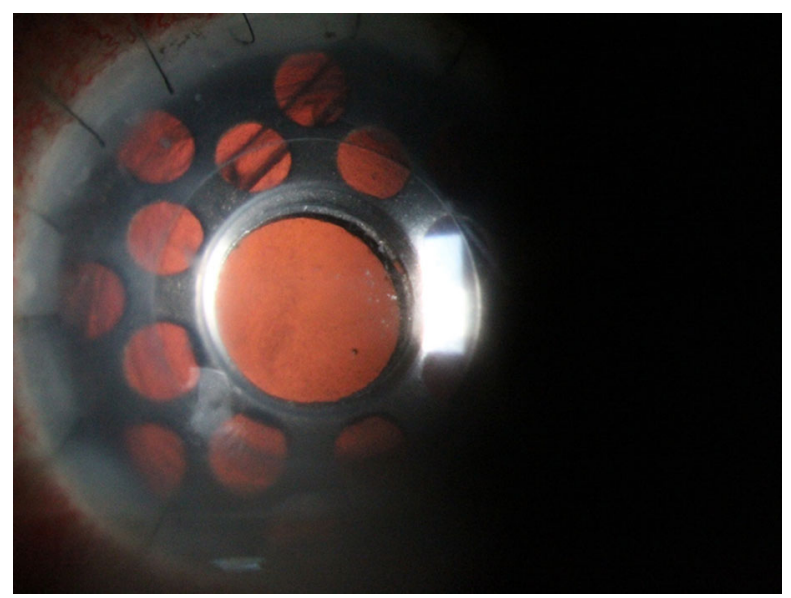

Fig. 4 Placement of tube tip behind the backplate of the keratoprosthesis

chronic and subacute inflammation contribute to an accumulation of inflammatory debris in the eye, leading to the occurrence of tube blockage during the early or late postoperative course. When the tube tip was positioned behind the backplate (Fig. 4), it was difficult to observe tube obstruction or perform Nd:YAG laser treatment. Therefore, we recommend that the tube be cut so that it is long enough to be seen through the KPro optic. However, it was difficult to place the tube tip just at the edge of the 3.0-mm optic of the KPro during anterior vitrectomy surgery when the IOP was not stable. In case 3, using the 3-port pars plana vitrectomy and stabilized IOP, the tube tip was much easier to place at the posterior edge of the optic.

In conclusion, blockage of tube orifices may occur in patients with KPro and GDD implantation. Treatment with Nd:YAG laser pulses could be a useful option to reopen the blocked tube in such patients. Placement of the tube tip at the edge of the optic enables laser intervention.

\section{ACKNOWLEDGEMENTS}

Funding. The study was performed at Zhongshan Ophthalmic Centre, Sun Yat-sen University, Guangzhou, China. No funding was received for the conduct of the study. Article processing charges were funded through Zhongshan Ophthalmic Centre.

Editorial Assistance. Editorial assistance in the preparation of this article was provided by AJE Company and funded by Zhongshan Ophthalmic Centre.

Authorship. All named authors meet the International Committee of Medical Journal Editors (ICMJE) criteria for authorship for this manuscript, take responsibility for the integrity of the work as a whole, and have given final approval to the version to be published.

Disclosures. Jianjun Gu, Yuying Zhang, Jiajie Zhai, Zhancong $\mathrm{Ou}$, and Jiaqi Chen have nothing to disclose.

Compliance with Ethics Guidelines. The retrospective analysis was approved by the institutional ethical committee of Zhongshan Ophthalmic Centre. All procedures followed were in accordance with the Declaration of Helsinki of 1964, as revised in 2013. All patients provided written informed consent for publication of clinical data.

Open Access. This article is distributed under the terms of the Creative Commons Attribution-NonCommercial 4.0 International License (http://creativecommons.org/licenses/ by-nc/4.0/), which permits any noncommercial use, distribution, and reproduction in any medium, provided you give appropriate credit to the original author(s) and the source, provide a link to the Creative Commons license, and indicate if changes were made.

\section{REFERENCES}

1. Banitt M. Evaluation and management of glaucoma after keratoprosthesis. Curr Opin Ophthalmol. 2011;22(2):133-6.

2. Vajaranant TS, Blair MP, McMahon T, Wilensky JT, de la Cruz J. Special considerations for pars plana tubeshunt placement in Boston type 1 keratoprosthesis. Arch Ophthalmol. 2010;128(11):1480-2. 
3. Tsai JH, Derby E, Holland EJ, Khatana AK. Incidence and prevalence of glaucoma in severe ocular surface disease. Cornea. 2006;25(5):530-2.

4. Lin MP, Ekşioğlu Ü, Mudumbai RC, Slabaugh MA, Chen PP. Glaucoma in patients with ocular chemical burns. Am J Ophthalmol. 2012;154(3):481-5.

5. Cade F, Grosskreutz CL, Tauber A, Dohlman CH. Glaucoma in eyes with severe chemical burn, before and after keratoprosthesis. Cornea. 2011;30(12):1322-7.
6. Lenis TL, Chiu SY, Law SK, Yu F, Aldave AJ. Safety of concurrent Boston type I keratoprosthesis and glaucoma drainage device implantation. Ophthalmology. 2017;124(1):12-9.

7. Netland PA, Terada H, Dohlman CH. Glaucoma associated with keratoprosthesis. Ophthalmology. 1998;105(4):751-7. 